Bacterial infections are common in cirrhotic patients as a result of defects in their immune defense system [1]. We report two patients with cirrhosis who developed meningitis following upper endoscopy.

A 41-year-old man with a history of hepatitis C-related post-hepatitic cirrhosis was admitted to our intensive care unit because of hematemesis. The patient underwent an emergency upper endoscopy, which showed esophageal varices. He was treated by ligation of the varices, and intravenous antibiotic prophylaxis with ceftriaxone was started. Twelve hours later, the patient suddenly became drowsy, his body temperature rose to $40^{\circ} \mathrm{C}$, and he then had a generalized seizure. Cerebral computed tomography (CT) was normal. A lumbar puncture revealed purulent cerebrospinal fluid (CSF). CSF antigen tests and cultures were negative. Ceftriaxone and amoxicillin were administered intravenously for 2 weeks. The patient was discharged from hospital without any neurological sequelae.

A 58-year-old man was admitted with Child B alcoholic cirrhosis. Four days after an upper endoscopy was performed he became confused and his temperature rose to $40^{\circ} \mathrm{C}$. Urinary bacteriological analysis and chest radiography were normal, and empirical treatment with piperacillin and ciprofloxacin was started. His neurological condition deteriorated and he was admitted into the intensive care unit. A cerebral CT scan was normal, but lumbar puncture revealed purulent CSF. CSF cultures and antigen tests were negative, but three blood cultures were positive for Streptococcus oralis. The patient was treated with amoxicillin. There were no signs of endocarditis on echocardiography. A dental CT scan showed that he had several dental abscesses. The patient died of septic shock and multiorgan failure.

\title{
Bacterial meningitis following upper gastrointestinal endoscopy in patients with cirrhosis - bear it in mind
}

Although bacterial meningitis seems to be rare in cirrhosis [2], previous reports have suggested a causal relationship between meningitis and upper gastrointestinal endoscopy [3]. The diagnosis of meningitis in cirrhosis can be difficult for several reasons: the clinical presentation of meningitis in cirrhosis has special characteristics, such as the absence of nuchal rigidity; the condition frequently presents as a febrile coma [1]; and drowsiness or confusion can be masked by the encephalopathy. In addition, cultures can be negative after antibiotic prophylaxis [4], as in our first case.

Upper endoscopy requires manipulation of the oropharyngeal areas, which can damage the oral mucosa, and be followed by transient bacteremia (with commensal organisms) in $2.5 \%$ of cases, as in the second case. Alcoholic patients often have poor oral hygiene, and any septic foci should be treated before upper endoscopy is performed [5].

Endoscopy_UCTN_Code_CPL_1AH_2AB

\section{Legrand', J. Denis², F. Drouhin²,} L. Lecuyer ${ }^{1}$, R. Plat ${ }^{1}$, A. Van De Louw ${ }^{1}$, S. Thierry ${ }^{1}$

${ }^{1}$ Intensive Care Unit, Centre Hospitalier

Sud-Francilien, Evry, France

${ }^{2}$ Hepatogastroenterology Unit, Centre Hospitalier Sud-Francilien, Evry, France.

\section{References}

${ }^{1}$ Pauwels A, Pines E, Abboura M et al. Bacterial meningitis in cirrhosis: review of 16 cases. J Hepatol 1991; 21: 830-834

2 Borzio M, Salerno F, Piantoni L et al. Bacterial infection in patients with advanced cirrhosis: a multicentre prospective study. Digest Liver Dis 2001; 33: 41 - 48

${ }^{3}$ Toyoda K, Saku Y, Sadoshima S, Fujishima M. Purulent meningitis after endoscopic injection sclerotherapy for esophageal varices. Intern Med 1994; 33: 706 - 709

${ }^{4}$ Garcia-Tsao G. Bacterial infections in cirrhosis: treatment and prophylaxis. J Hepatol 2005; 42: S85-S92

${ }^{5}$ Novacek G, Plachetzky U, Potzi R et al. Dental and periodontal disease in patients with cirrhosis: role of etiology of liver disease. J Hepatol 1995; 22: $576-582$

\section{Corresponding author}

\section{Legrand, MD}

Centre Hospitalier Sud Francilien Site Evry - Quartier du Canal 91014 Evry

France

Fax: $\quad+33-1-60-87-52-29$

Email: m.legrand@libertysurf.fr 